\title{
Narrow funnel-like interaction energy distribution is an indicator of specific protein interaction partner.
}

Juyoung Choi ( $\nabla$ rgdfs@sogang.ac.kr)

Sogang University https://orcid.org/0000-0002-4290-998X

\section{Article}

Keywords: protein-protein interaction, kinases, E3 ubiquitin ligases, protein docking, interaction energy

Posted Date: July 25th, 2022

DOI: https://doi.org/10.21203/rs.3.rs-1412786/v3

License: (c) (i) This work is licensed under a Creative Commons Attribution 4.0 International License. Read Full License 
Title

- Full title: Narrow funnel-like interaction energy distribution is an indicator of specific protein interaction partner.

- Short title: Protein interaction prediction using energy analysis

\author{
Authors \\ Juyoung Choi ${ }^{1 *}$
}

\begin{abstract}
Protein interaction networks underlie countless biological mechanisms. However, most protein interaction predictions are based on interspecies biological evidence (knowledge-based predictions) that are biased to well-known protein interaction or physical evidence that exhibits low accuracy for weak interactions and requires high computational power. In this study, a novel method has been suggested to predict protein interactions using interaction energy distribution. Protein interactions with specific partners can be predicted by investigating narrow funnel-like interaction energy distribution, exhibiting a stable state in a specific orientation. In this study, it was demonstrated that various protein interactions with specific interaction partner, such as protein interaction with kinase and E3 ubiquitin ligases, have narrow funnel-like interaction energy distribution. Moreover, algorithm and deep learning model for prediction of general protein interaction partner and substrate of kinase and E3 ubiquitin ligase were developed. The prediction accuracy of this method is similar to or even better than that of yeast two-hybrid screening, which is the most widely applied interaction screening method. Ultimately, this knowledge-free protein interaction prediction method will broaden our understanding of protein interaction networks.
\end{abstract}

\title{
Teaser
}

A novel method of determining specific protein interactions was developed by investigating narrow funnel-like interaction energy distribution.

\section{MAIN TEXT}

\section{Introduction}


Most biological activities occur through complex biomolecular interaction networks. After the genome sequencing of several tens of thousands of organisms, understanding biomolecule networks beyond the individual genes is becoming increasingly important. In particular, proteinprotein interactions (PPI) are among the most diverse biomolecular networks and the center of system biology. Several experimental techniques, such as yeast two-hybrid screening, coimmunoprecipitation, affinity-chromatography, tandem affinity purification-mass spectroscopy, etc., have been developed to identify PPI (1). However, with the rapid advancement of proteome identification, experimental results cannot keep up with the potential number of protein combinations. Recently, 200 million proteins in various organisms were predicted or identified (2). Meanwhile, the international consortium curated only approximately 1 million PPIs from literatures and datasets $(3,4)$. Moreover, among the identified PPI, approximately $70 \%$ are Homo sapiens-related (4). Most PPI might still be unidentified. Therefore, in silico methods, as well as high throughput experimental approaches, have been developed to broaden our PPI-related knowledge (5).

In silico PPI prediction methods utilize biological, structural and physical results. Biological evidence includes gene or domain fusion $(6,7)$, gene neighborhood $(6,8)$, interolog (9), coexpression $(6,10)$, coevolution $(11)$, and phylogenetic similarity $(6,12)$. Although biological result-based PPI predictions have a powerful predictive ability, in addition to being applicable on a large scale, they are highly biased to well-known PPI. Except for PPIs with rare molecular features (gene or domain fusion and gene neighborhood), omics scale interspecies data are required or at least similar protein interactions in other species should be identified to predict PPI using biological results $(5,13)$. Structural and physical evidence-based PPI prediction utilizes protein-protein interface templates $(14)$, interaction energy $(13,15)$, and shape complementarity $(15,16)$. Template-based PPI predictions require known PPIs with similar interface structures (14, 17). All these knowledge-based PPI prediction are not enough to discover new PPI, since most PPI might still be unknown. Conversely, PPI predictions with interaction energy and shape complementarity are independent of accumulated large-scale data and known PPIs. However, shape complementarity is different for each PPI type. Obligate PPIs, which are stable only when interacting with each other, have higher shape complementarity, although transient PPIs exhibit low shape complementarity $(5,18)$. Moreover, PPI prediction using interaction energy require a lot of computational power and is not suitable for large scale prediction. Especially, protein docking programs, which calculate the possible direction of interaction and interaction energy, have a high computational cost $(5,15)$. Transient PPIs with weak interactions are difficult to 
predict using interaction energy (15). However, most PPIs are weak, and weak PPIs play a pivotal role in protein interaction networks (19). Therefore, high computational cost and the inability to predict transient PPIs with weak interactions represent the major drawbacks of protein interaction partner prediction using in silico protein docking.

One of the most difficult PPIs to predict is that of enzyme-specific interaction partners, such as substrates, inhibitors, and regulators, or signaling pathway interactions. Their diversity of complicated signaling pathways (20) makes biological evidence- and structural template-based PPI prediction difficult. Furthermore, due to weak and transient interaction properties, interaction energy and shape complementarity are not useful for the prediction of these PPIs $(15,18)$. However, understanding PPIs in signaling pathways, such as the interactions of kinases or E3 ubiquitin ligase (EUL) with their interaction partners, is one of the most crucial parts of biology. In mammals, kinases and ubiquitination-related enzymes are the most and the fifth most abundant enzymes in signaling pathways, respectively (20). Moreover, in plants, approximately $5 \%$ of the protein-coding genes in rice and Arabidopsis encode kinases and EULs (21-23). Despite the importance of kinases and EULs, most interaction partner predictions of kinases and EULs are mostly based on biological evidence, which require identified PPI with similar amino acid sequences or domains (24-30). Moreover, because of the lack of identified PPIs, most prediction tools are limited to human kinases or EULs (24, 26-30). Therefore, in most cases, no highaccuracy PPI prediction tools are available for kinases and EULs.

In this study, I suggest a new, interaction energy distribution-based PPI prediction strategy. PPIs with a specific interaction partner, such as PPIs of kinase and EULs, exhibit the specificity to maintain sophisticated signaling pathways. For instance, in humans, kinase phosphorylates specific phosphorylation sites ranging from one to hundreds among approximately 700,000 potential phosphorylation sites (31). Moreover, most regulators and inhibitors act when they interact with kinases only in a specific orientation $(32,33)$. Although these PPIs are weak, the specificity of these interactions observed in a specific orientation distinguishes them from non-interacting protein pairs $(31,34)$. Based on this specificity, it was hypothesized that an interaction energy diagram of PPI with specific interaction partners would display a narrow funnel-like landscape, exhibiting a stable state in a specific orientation (Fig. 1a). In this study, using Rosetta energy function, knowledge (or Boltzmann relation)-based macromolecular energy function (35), the PPI interaction energy distribution was calculated.

Although there are considerable differences of interaction energy distributions between interacting and non-interacting protein complexes, setting a classification criterion with high accuracy is difficult. Recent advances in deep learning have made it possible to extract hidden 
107 features from complicated data and classify them. (36) Training using interaction energy

108 distribution data of interacting and non-interacting protein complex can be employed to classify

109 the interaction energy distribution of unknown protein pairs into interacting or non-interacting

110 protein interaction energy distribution. This binary classification problem has been successfully

111 resolved in various field using machine learning (37). In this study, deep learning models were

112 developed to predict general protein interaction partners and substrates of kinase and E3 ubiquitin

113 ligase.

\section{Results}

\section{Workflow for the interaction energy distribution of kinases/E3 ubiquitin ligases and}

\section{their interaction partners}

To analyze the interaction energy landscape of kinases/EULs and their interaction partners, I retrieved 135 kinase-interaction partners, including regulator, inhibitor, and substrate, and 189 EUL-substrate pairs from known databases (3, 38, 39). To construct negative controls, I randomly paired kinases and EULs with different partners with no evidence of the existence of functional interactions in these pairs. Randomly paired partners might later turn out to be real interaction partners, but most likely they would not be interaction partners. Therefore, as a negative control, 106 kinase- and 124 EUL-random partner pairs were constructed (Fig. 1B, Supplementary Data 1). Using Phyre2, a server for template-based structure modeling (40), full predicted structures of kinases, EULs, and their partners were obtained (Supplementary Data 2). Because of computational costs, the calculation of every possible protein docking structure is nearly impossible. Therefore, 1,000 possible docking structures per pair were sampled using two docking programs (Table 1, Fig. 1B). RosettaDock uses the Monte-Carlo algorithm with a knowledge-based energy function (35) and includes the structure refinement of the docking structures (41). 
HDOCKlite uses the fast Fourier transform (FFT) algorithm with a shape-based scoring function and does not include structure refinement (42).

To analyze the interaction energy landscape (Fig. 1A), I calculated the interaction energy with Rosetta energy and the following equation:

$$
\begin{gathered}
\text { Interaction Energy }=\{\text { Rosetta energy of simulated docking structure }\} \\
-\sum_{\text {Each component in pair }}\{\text { Rosetta energy of single components }\}
\end{gathered}
$$

As every theoretical protein energy exhibits differences compared with the experimental data, I ignored energy change because of structure change and considered only the affinity to minimize error. To indicate interacting positions, I used Interface Root Mean Square deviation (iRMS, 43). Originally, iRMS was developed to compare simulated docking and native structures (44). In this study, I slightly changed iRMS. Instead, of the native structure, I set the strongest interacting structure as a reference structure (Fig. 1C). The redefined iRMS was as follows:

$$
i R M S=\sqrt{\frac{1}{N} \sum_{i=1}^{N}\left\|x_{i}-y_{i}\right\|^{2}}
$$

\section{$N$ : the number of backbone atom of interface residues}

$x_{i}$ : the position of $i$ th backbone atom of interface residues in docking structure $y_{i}$ : the position of $i$ th backbone atom of interface residues in the strongest interacting structure

As Fig. 1C shows, in the strongest interacting structure, residues closely located within $10 \AA$ were set as interface residues. In different docking structures, the same residues were in different positions. iRMS is the root mean square deviation of the backbone atoms of interface residues in two structures. For every docking structure generated from each enzyme-interaction and random partner pairs using two docking programs, interaction energy and iRMS were analyzed and plotted (Supplementary Data 3 and Supplementary Data Figs 1-4: generated using RosettaDock; Supplementary Data 4 and Supplementary Data Figs 5-8: generated using HDOCKlite). As HDOCKlite does not include structure refinements, the interaction energy calculation indicates that 
most docking structures with HDOCKlite were unfavorable (Interaction energy $>0 \mathrm{kcal} / \mathrm{mol}$ ). However, affinity comparison would be possible by comparing the interaction energies.

\section{Narrow funnel-like interaction energy distribution of kinases/EULs and their specific interaction partners}

To illustrate the structural meaning of the narrow funnel-like interaction energy distribution, Fig. 2 shows the interactions between cyclin-dependent kinase 4 (CDK4) and its specific/random partners. It is well-known that CDK4 interacts with G1/S-specific cyclin-D1 (CCND1) and mediates cell cycle progression (G1-to-S phase, (45). Inhibitor of nuclear factor kappa-B kinase subunit alpha (IKK $\alpha$ ) is part of the IKK complex and phosphorylates inhibitor of nuclear factor kappa-B (46). Then, the activated nuclear factor kappa-B mediates immune response, inflammation, and apoptosis (47). CDK4 randomly paired with IKK $\alpha$ and there is no literature and dataset have identified that they form a functional PPI. Using RosettaDock, 1,000 docking structures of CDK4-CCND1 and CDK4-IKK $\alpha$ pairs were established. Then, by calculating the interaction energy, the top five strongest docking structures have been summarized in Fig. 2. The CDK4-CCND1 pair, an identified interaction pair, interacts in similar positions. However, the CDK4-IKK $\alpha$ pair, a random pair, shows scattered interacting positions on the surface of CDK4 and IKK $\alpha$. Although the average interaction energies are similar and a little higher in the CDK4-IKK $\alpha$ pair, the average iRMSs of CDK4-CCND1 (functionally interacting pair) complex was significantly smaller than CDK4-IKK $\alpha$ (random pair).

For the quantitative analysis of the interaction energy distribution, I devised a strategy to distinguish narrow funnel-like interaction energy distribution (Fig. 3A). Docking structures with weak interaction are regarded as functionally non-interacting structure. Docking structures with strong interaction but large iRMS indicate broad funnel-like interaction energy distribution. Therefore, I only had to consider docking structure ratios with strong interactions and small iRMS. I defined a score (narrow funnel distribution) for the distinction of narrow funnel-like distribution (Fig. 3A). If the narrow funnel distribution was bigger than or equal to certain distribution criteria, this interaction energy distribution was determined as exhibiting narrow funnel-like distribution. Then, only three criteria (Interaction energy, iRMS, and distribution criteria) had to be decided (Fig. $3 \mathrm{~A})$.

As per my hypothesis, if a protein pair with specific interacting partners showed narrow funnel-like interaction energy distribution, specific partners could be predicted with interaction energy distribution analysis by taking specific criteria. Therefore, I calculated the difference between the true-positive (identified pair with narrow funnel-like interaction energy distribution) 
and false-positive (random pair with narrow funnel-like interaction energy distribution) ratios for each criterion. As per the hypothesis, the narrow funnel distribution of the functionally interacting protein complex was higher than that of random protein pairs in the case of most criteria.

For whole protein pairs, docking structures generated by RosettaDock, HDOCKlite showed up to $11.9 \%-20.3 \%$ difference of true- and false positives (Supplementary Data Fig. 9). Interaction energy, iRMS, and distribution criteria at the maximum points are described in Supplementary Table 1. As the interaction calculation depends on structure accuracy, I filtered out low-accuracypredicted structures with less than $70 \%$ region with high confidence $(>90 \%$ confidence, $(40)$ and analyzed the remaining pairs with high-accuracy structure prediction (identified kinase-interaction pair: $\mathrm{n}=56$ for RosettaDock, $\mathrm{n}=58$ for HDOCKlite; random kinase pair: $\mathrm{n}=52$ for RosettaDock, $\mathrm{n}=54$ for HDOCKlite; identified EUL pair: $\mathrm{n}=59$ for RosettaDock, $\mathrm{n}=70$ for HDOCKlite; random EUL pair: $\mathrm{n}=62$ for RosettaDock and HDOCKlite). The docking structures, generated by RosettaDock and HDOCKlite, showed up to $17.9 \%-26.4 \%$ difference of true- and false positives (Supplementary Data Fig. 10). Interaction energy, iRMS, and distribution criteria at the maximum point are described in Supplementary Table 2.

As protein global docking works well for small proteins (48), I again filtered out pairs with large proteins (>700 residues) and analyzed the remaining pairs with high-accuracy structure prediction and relatively small proteins (identified kinase-interaction pair: $\mathrm{n}=28$ for RosettaDock and HDOCKlite; random kinase pair: $n=26$ for RosettaDock and HDOCKlite; identified EUL pair: $\mathrm{n}=25$ for RosettaDock, $\mathrm{n}=36$ for HDOCKlite; random EUL pair: $\mathrm{n}=28$ for RosettaDock and HDOCKlite). For the kinase pairs, the docking structures generated by RosettaDock and HDOCKlite showed up to $44.2 \%$ and $48.9 \%$ difference of true- and false positives, respectively. For the EUL pairs, the docking structures generated by RosettaDock and HDOCKlite showed up to $35.9 \%$ and $27 \%$ difference of true- and false positives, respectively (Fig. 3B). Interaction energy, iRMS, and distribution criteria at the maximum points are described in Table 2 . In order to investigate the effectiveness of finding specific interaction partners using interaction energy distribution, true- and false-positive rates were compared with those of the yeast two-hybrid screening approach. True- and false-positive rates from genome-scale yeast two-hybrid trials in three species were compared (49). Whole pairs and pairs with high-accuracy structure predictions had similar accuracy to those of the yeast two-hybrid screening (Supplementary Data Figs 9 and 10). Pairs with high-accuracy structure predictions and relatively small proteins showed 
significantly better results than those of the yeast two-hybrid screening approach (Fig. 3C). Interaction energy, iRMS, and distribution criteria at the maximum points are described in Table 2.

\section{Interaction energy distribution of other protein complexes}

As kinase and EUL PPIs represent only a small part of the whole PPIs, I investigated the interaction energy distribution in other protein complexes. I retrieved 183 curated experimentally determined protein complex structures from the IntAct database (4), then analyzed the PPI between the interacting chains with the interface area in the protein structure using RosettaDock (see Fig. $1 \mathrm{~B}$ and Supplementary Data 5). The interaction energy and iRMS distribution of the protein complexes are plotted in Supplementary Data Fig. 11. Moreover, I retrieved 110 experimentally determined protein structures, which were unlikely engaged in direct interactions from the Negatome 2.0 database as negative controls (50), then analyzed the PPI using RosettaDock (see Fig. 1B and Supplementary Data 5). The interaction energy and iRMS distribution of the protein complexes are plotted in Supplementary Data Fig. 12.

To confirm whether other protein complexes showed narrow funnel-like interaction energy distribution, I calculated the iRMS averages of simulated docking structures. As per the hypothesis (Fig. 1A), the average iRMS of the interacting protein complexes were significantly smaller than the average iRMS of non-interacting protein pairs $(95 \%$ confidence using Student's t-test, Fig. 4). Furthermore, I functionally annotated these proteins according to level-2 gene ontology distribution from Generic GO slim (51), then divided the protein pairs according to the related biological processes and calculated the average iRMS for each part (Fig. 4). Almost every division showed smaller average iRMS of the interacting complexes than those of the non-interacting pairs, except for the negative regulation of the biological processes. In particular, protein pairs related to cellular, metabolic, and developmental processes, signaling, response to stimuli, and biological process regulation showed significantly smaller average iRMSs than non-interacting protein pairs (95\% confidence using Student's t-test). These processes are well-known to display molecular cascades with specific PPIs. Among those, cellular process-, developmental process-, and response to stimuli-related proteins pairs showed higher differences (99\% confidence using Student's t-test). In particular, developmental process-related proteins pairs showed the biggest differences of iRMSs between the interacting complexes and non-interacting pairs (median of average iRMS of the interacting complexes: $22.37 \AA$, median of average iRMS of non-interacting pairs: $29.6 \AA$ ). This result might be related to developmental processes controlled by complex molecular cascades and networks with specific PPIs (52). 


\section{Deep learning model to predict protein interaction partners}

As I have heretofore demonstrated, there are significant differences in the interaction energy distribution between interacting and non-interacting protein complexes. Thus, I developed deep learning models to predict protein interactions based on complex interaction energy distributions. As described in Fig. 5, the prediction program trained with interaction energy distributions of interacting and non-interacting protein pairs uses deep learning to extract hidden distinguishable features from complex distribution patterns and classify them. Then, based on this interaction energy distribution, the prediction program predicts whether the given protein pair will interact.

To develop the prediction model, protein interaction energy distributions of 183 experimentally determined interacting protein complex from the IntAct database (4) and 110 noninteracting protein pairs from the Negatome 2.0 database (50) were used for training (Supplementary Data 5). Additionally, I tested seven optimizers from the Keras library, including Adam, SGD, RMSprop, Adadelta, Adamax, Nadam, and Ftrl (53), to construct the optimal deep learning model for interaction prediction. Moreover, considering the amount of input data and the findings of previous studies $(54,55)$, I constructed seven candidate layer structures and tested each. Each of the 49 deep learning models was analyzed with 1-200 epochs (Supplementary Fig. 13). The optimal model for prediction showed an accuracy of $77.6 \%$ by utilizing the Adam optimizer containing a 1024-64-2-layer structure and 109 epochs (Table. 3). To assess the predictive performance of this deep learning model, the receiver operating characteristic (ROC) curve from 10-fold cross validation with data shuffling was analyzed. The area under curve (AUC) of this model was 0.834 (Fig. 6), demonstrating a higher predictive performance than that of yeast two-hybrid screening (Fig. 3C).

To apply this deep learning method to predict substrates of EULs and kinases, I retrieved EULs and kinases from various species along with information about their substrates from the Ubibrowser2.0 (25) and PhosphoSitePlus (56) databases. Then, I retrieved predicted structures in the AlphaFold database (57) with less than 700 residues and more than $70 \%$ prediction confidence in at least half the region. In total, 63 EULs and their substrates and 214 kinases and their substrates were retrieved (Supplementary Data 7). I then randomly paired enzymes and substrates as a negative control. Among these, pairs with evidence of interactions in the IntAct (4) or STRING databases ( 6 ) were filtered out. A total of 63 EUL-random substrate pairs and 203 kinase-random substrate pairs were constructed. Their interaction energy distribution was then analyzed using RosettaDock (41) and HDOCKlite (42), as shown in Fig. 1. To construct an optimal deep learning model for interaction prediction, 49 candidate models with 7 optimizers in 
the Keras library and 7 candidate layer structures were analyzed with 1-100 epochs for EULs and 1-50 epochs for kinases (Supplementary Fig.14-16). The deep learning model trained using the RosettaDock data (Supplementary Figs. 14 and 15) performed better than that trained using the HDOCKlite data (Supplementary Fig.16). Therefore, only the deep learning models trained using the RosettaDock data were further analyzed.

For substrate prediction of EULs, the optimal prediction model showed a 77.7\% accuracy using an Adam optimizer containing a 1024-2-layer structure and 61 epochs. For substrate prediction of kinases, the optimal model for prediction showed a $61.4 \%$ of accuracy with an Adam optimizer containing a 1024-64-2-layer structure and 9 epochs (Table. 3). ROC curves obtained from 10-fold cross validation with data shuffling were then analyzed to test the predictive performance of these deep learning models. The AUCs of these models were 0.711 and 0.601 for EULs and kinases, respectively (Fig. 6). These result demonstrates higher and similar predictive performance than that of yeast two-hybrid screening, respectively (Fig.6 and Fig. 3C).

\section{Discussion}

In this study, I demonstrated that the interaction of a protein with a specific interaction partner has a narrow, funnel-like interaction energy distribution. In addition, interaction energy distributions between interacting and non-interacting protein pairs are substantially different. Especially, PPIs related with various signaling pathway showed much narrower interaction energy distribution than those of random pair. Although these PPIs, including PPIs of kinases or EULs, are difficult to predict and validate using experiments and previous prediction methods, the interaction energy distributions had considerably distinguishable traits. I then developed a deep learning model to predict interactions based on the interaction energy distribution. ROC curve analysis showed that general protein interaction prediction and EUL substrate prediction had a good predictive performance, with an AUC of 0.7-0.9. However, kinase substrate prediction only had an acceptable predictive performance, with an AUC of approximately 0.6. For interaction energy distribution analysis, I used whole interactions of kinases, including regulators and substrates. However, when I narrowed those to interactions with only substrates, the weakness of interactions might hamper the prediction. Thus, for further improvement and development of this prediction model, a bigger training data set may be essential. Finally, the deep learning model trained using RosettaDock data performed better than that trained using HDOCKlite data, indicating that a more accurate interaction energy calculation with structure refinement is necessary to accurately predict protein interactions. 
Currently, most protein interaction prediction methods are biological evidence- and known protein complex structure template-based $(5-11,14,17)$. However, these methods that require omics scale interspecies biological data or known similar interactions display limited predictability. Knowledge-free protein interaction prediction methods would be necessary to describe the interactome in various species. Therefore, I focused on a protein docking program, which simulates the physical interactions of proteins. Protein docking programs have been developed for over 30 years (58). With communitywide docking program assessment, CAPRI (Critical Assessment of PRediction of Interactions, 59), recent docking programs are showing good performance in predicting protein complex structures (60). However, docking programs are rarely used to find interacting partners for two reasons: low accuracy in weak and transient interactions and computational costs $(5,15,58)$.

In 2011, using supercomputers, protein docking structures of interacting and noninteracting protein pairs were generated for up to 100,000 structures per each pair and compared (15). Although over half of the protein complexes showed a better docking score than $85 \%$ of the background, the approach showed lower performance for enzyme-inhibitor interactions. Moreover, most protease interactions were similar to those of the background (15). Three reasons explain why docking score, consisting of interaction energy and the shape complementarity of the docking structure, was not enough for interacting partner prediction of high accuracy. First, investigation of proteomic quantity is missing. Even a interaction partner with weak interaction energy can be a dominant interaction partner if its cellular amount is large (19). However, most cellular quantities of proteins are unknown. Second, all protein energy functions exhibit a small difference from reality. Even though multiple protein energy functions were developed, energybased free protein structure modeling showed poorer performance than template-based modeling (61). Moreover, the Rosetta energy function, one of the successful and widely used energy functions, predicts that the $\Delta \Delta \mathrm{G}$ of the HIV1 protease T193V mutation is $-4.95 \mathrm{kcal} / \mathrm{mol}$, but the experimental result was $-1.11 \mathrm{kcal} / \mathrm{mol}(35,62)$. In contrast, due to the competition effect, a slightly higher interaction energy is enough to be a dominant interaction partner (31). Therefore, minor errors in the energy function can hinder the interaction partner prediction. Third, low shape complementarity of transient interaction may not be distinguishable from those of others $(5,18)$. Certain docking scores use protein complex geometric shape complementarity $(42,63)$, but transient interaction partners do not have dominant shape complementarity, distinguishable from those of others.

In this study, I used specificity, as well as interaction energy, to distinguish protein interaction partners. Specificity is the result of a long evolution (64). Because of specificity, 
complicated molecular cascades in biological processes and complex life activities can be maintained (31). As specificity in multiple PPIs depends on the interaction in specific regions, such as Vander Waals and electrostatic interactions $(31,64)$, such specific PPIs are in a specific orientation. For example, p53, a well-known tumor suppressor, has more than 20 and 10 phosphorylation and ubiquitination sites, respectively. Each site is phosphorylated and ubiquitinated by specific kinases and EULs (65). These PPIs in specific orientation result in narrow funnel-like interaction energy distribution (Fig. 1A). In this study, I showed that specific interaction partner search with narrow funnel-like interaction energy distribution achieved similar accuracy as yeast two-hybrid screening (Fig. 3C and Fig. 6). Therefore, the narrow funnel-like interaction energy distribution is a key indicator of specific interaction partners.

High computational cost is another hurdle to using docking programs for interaction partner predictions. In this study, PPI prediction with narrow funnel interaction energy distribution using data from HDOCKlite, an FFT-based rigid body docking program (42), showed good performance in specific interaction partner distinction (Fig. 3C). As this program runs fast even on personal computers (Table 1), it can predict specific interaction partners without additional computational power. Moreover, protein interaction prediction on the proteomic level might be feasible with small computational power. RosettaDock includes docking structure refinement using the Monte-Carlo algorithm (41). Therefore, it can calculate cooperative interactions, accompanied by structure changes (66). Furthermore, I showed that 1,000 docking structures per each pair are enough for interaction partner prediction. Therefore, the narrow funnel-like interaction energy distribution-based approach adapted to small computational power. Due to the small computation amount, interaction predictions on the proteome scale are also possible.

However, this study also has clear limitations. First, protein structures should be predicted or determined and the structure prediction result affects PPI prediction results (Fig. 3, Supplementary Data Figs 9 and 10). However, amazing progress has been recently achieved in protein structure prediction. Protein structure prediction tools using deep learning were developed $(67,68)$. In a recent blind protein structure prediction assessment, Critical Assessment of protein Structure Prediction 14 (61), AlphaFold predicted certain targets with higher accuracy than template-based predictions (69). Protein structure prediction error is decreasing close to the error of protein structure determination using X-ray crystallography $(68,69)$. Moreover, predicted structures are being accumulated in databases and such structures are being published (57). Therefore, proteome-scale predicted protein structures can be used by anyone in the near future. As I used template-based structure prediction, this study is not fully template-free. However, 
using protein structure predicted using deep learning, knowledge-free protein interaction predictions could be achieved.

Second, protein size affects the prediction results (Fig. 3, Supplementary Data Figs 9 and 10). As global docking works better for small than large proteins (48), interaction energy distribution is affected by protein size. This bias can be improved through high resolution in silico protein docking with more docking structures (15). Furthermore, interaction partner prediction with interaction energy distribution can be used in parallel with other interaction partner predictions. Widely used biological result- and template structure-based protein interaction prediction methods are biased by well-studied interaction pairs (5). However, the interaction energy distribution is completely irrelevant for the extent of how well-studied these interactions are. This method is biased by protein pairs with well-predicted structures and of small size (Fig. 3, Supplementary Data Figs 9 and 10). They can be used together to complement each other.

To improve this study further, improvement of protein energy function should be considered. There are two kinds of protein energy functions. One is the physics-based energy function, constructed based on classical mechanics and correction terms with perturbations in quantum mechanics, such as the CHARMM force field (70). The other is knowledge-based energy function, using Boltzmann relation to retrieve energy terms from experimentally determined protein structures, such as Rosetta energy (35). These energy functions are developed using a bottom-up approach consisting of each formula and parameter of physical properties like building blocks $(35,70)$. Terms might also be missing or contain big errors. Therefore, from a holistic point of view, protein energy might be retrieved using the Boltzmann relation from protein structures. To do so, significant amounts of structures are necessary, although the experimentally identified protein structures are limited. However, due to the recent progress in the protein structure prediction-related deep learning field, we can predict distribution of protein structures. In AlphaFold, predicted distance and torsion distribution are generated by deep learning and the potentials are retrieved with predicted distributions using Boltzmann relation without specific physical energy formula (67). Therefore, interaction energy can be generated with a top-down approach using deep learning. Moreover, the Boltzmann distribution is valid in the equilibrium state (71). However, living organisms are dynamic, open, and are in a nonequilibrium state. To describe life phenomena more precisely, nonequilibrium statistical mechanics, such as generalized Boltzmann distribution, would be necessary $(72,73)$.

Another possible interaction energy distribution analysis application is that narrow funnellike interaction energy distribution can be a specificity indicator. Finding specific interactions is important in drug discovery. Nonspecific interactions can result in side effects (74). Even though 
narrow funnel-like interaction energy distribution does not guarantee the exclusion of binding other molecules, it shows that proteins bind to a specific site and can be used as a specific interaction indicator. In particular, several kinases and EULs are considered as targets of multiple drugs, such as anticancer drugs $(75,76)$. Therefore, this study could be applied in drug discovery as a specific interaction indicator.

\section{Materials and Methods}

\section{Protein structure preparation}

To analyze kinase interactions, 135 kinase-interaction partner pairs were retrieved from kinase and protein interaction databases $(3,38)$. Using NumPy's random number generator (77), 106 random, not identified as interacting pairs in literatures and datasets, were generated (Supplementary Data 1). From the E3 ubiquitin ligase-substrate interaction database UbiBrowser (25), 189 E3 ubiquitin ligase-substrate interactions were retrieved. Using NumPy's random number generator (77), 124 random, not identified as interacting pairs in literatures and datasets, were generated (Supplementary Data 1). Using the Phyre2 server (40), protein structures were predicted with the intensive mode option (Supplementary Data 2).

To analyze interacting protein complexes, 183 protein complex structures were retrieved from the protein interactome database IntAct (4). Interacting chains in the protein complexes were analyzed using the interfaceResidue script of PyMOL (78). As negative controls, 110 experimentally determined protein structures, unlikely engaged in direct interactions, were retrieved from the Negatome 2.0 database (51).

\section{Protein docking structure generation using RosettaDock}

For RosettaDock, I used the Rosetta 2020.08 bundle (41). Each kinase and E3 ubiquitin ligase protein interaction pairs were merged using PyMOL (78). Kinase or E3 ubiquitin ligase chain IDs were designated as " $\mathrm{A}$ " and those of interacting partners as "B" using PyMOL (78). Before kinase and E3 ubiquitin ligase protein pair docking using RosettaDock (41), I optimized their side-chain conformations (prepacking) using the following command:

./bin/docking_protocol.static.linuxgccrelease -in:file:s (Merged protein structure file) docking:partners A_B -dock_pert 38 -randomize1 -randomize2 -spin -out:path:all (Output directory)

Next, I generated 1,000 docking structures using the following command:

./bin/docking_protocol.static.linuxgccrelease -in:file:s (prepacked files) -docking:partners A_B -dock_pert 38 -randomize1-randomize2 -spin -use_ellipsoidal_randomization true -nstruct 1000 -out:path:all (output directory)

To analyze interacting proteins complexes and non-interacting protein pairs, I optimized their side-chain conformations (prepacking) using the following command:

./bin/docking_protocol.static.linuxgccrelease -in:file:s (protein complex structure file) docking:partners (chain ID of proteins which participating in interaction)_(chain ID of proteins in 
opposite side of interaction) -dock_pert 38 -randomize1 -randomize2 -spin -out:path:all (output directory)

Then, I generated 1,000 docking structures using the following command:

./bin/docking_protocol.static.linuxgccrelease -in:file:s (protein complex structure file) docking:partners (chain ID of proteins which participating in interaction)_(chain ID of proteins in opposite side of interaction) -dock pert 38 -randomize1 -randomize2 -spin use_ellipsoidal_randomization true -nstruct 1000 -out:path:all (output directory)

To analyze kinase and E3 ubiquitin ligase interactions using HDOCKlite (42), I generated 1,000 docking structures using the following command:

./hdock (kinase or E3 ubiquitin ligase) (Interaction partner) -out (docking file)

./createpl (docking file) top1000.pdb -nmax 1000 -complex -models

All commands were written in a Linux shell file and executed.

For further analysis, kinase or E3 ubiquitin ligase chain IDs were designated as " $\mathrm{A}$ " and those of interacting partners as "B" using PyMOL (78). The graphical representations of the docking structure were exported using PyMOL (78).

\section{Interaction energy and iRMS analysis}

To calculate interaction energy, I calculated the Rosetta energy of each protein or complex using the following command: file)

./bin/score_jd2.linuxgccrelease -in:file:s (protein or complex) -out:file:scorefile (score

Then, I calculated the interaction energy using the following equation:

$$
\begin{gathered}
\text { Interaction Energy }=\{\text { Rosetta energy of simulated docking structure }\} \\
-\sum_{\text {Each component in pair }}\{\text { Rosetta energy of single components }\}
\end{gathered}
$$

Then, iRMS were calculated using DockQ (43) and the following command: file)

./DockQ.py (Docking structure) (Strongest interacting docking structure) -short $>>$ (iRMS

For interacting protein complexes and non-interacting protein pairs, iRMS were calculated using the following command:

./DockQ.py (Docking structure) (Strongest interacting docking structure) -short native_chain 1 (chain ID of proteins which participating in interaction) -model_chain1 (chain ID of proteins which participating in interaction) -native_chain2 (chain ID of proteins in opposite side of interaction) -model_chain2 (chain ID of proteins in opposite side of interaction) -perm1 perm2 $>$ (iRMS file) 
All commands were written in a Linux shell file and executed.

\section{Gene ontology analysis}

The bioinformatics analysis was performed as described previously (79), with small modifications. Briefly, using OmicsBox v2.0.36, protein sequences were blasted specifically using the NCBI nr database. Functional domains and motifs were distinguished using the EMBLEBI InterPro database (80). Then, OmicsBox was used to annotate these results. Finally, I summarized gene ontology results according to level-2 gene ontology distribution from Generic GO slim (51) using OmicsBox.

\section{Development of deep learning model and validation}

To construct training dataset for general PPI prediction using deep learning, iRMS and interaction energy of 183 protein complex structures, retrieved from IntAct database (4) and 110 non-interacting protein pairs, retrieved from the Negatome 2.0 database (51) were calculated as above. To construct training dataset for prediction of kinase and EUL substrate, 214 kinasesubstrate pair and 63 EUL-substrate pair were retrieved from PhosphoSitePlus (56) and UbiBrowser 2.0 (25) database, respectively (Supplementary data 7). Predicted protein structure were retrieved from AlphaFold database (57). Then, 1,000 simulated docking structure were generated using RosettaDock (41) and HDOCKlite (42), as described above. Then, interaction energy and iRMS were calculated as described above.

Deep learning model were constructed using Keras library (53). All data and codes, used for deep learning, are publicly available on GitHub (https://github.com/cjy318/protein_interaction). 
References

1. V. S. Rao, K. Srinivas, G. Sujini, G. Kumar, Protein-protein interaction detection: methods and analysis. International journal of proteomics 2014, (2014).

2. A. Bateman, M.-J. Martin, S. Orchard, M. Magrane, R. Agivetova, S. Ahmad, E. Alpi, E. H. Bowler-Barnett, R. Britto, B. Bursteinas, UniProt: the universal protein knowledgebase in 2021. Nucleic Acids Research, (2021).

3. S. Orchard, S. Kerrien, S. Abbani, B. Aranda, J. Bhate, S. Bidwell, A. Bridge, L. Briganti, F. S. Brinkman, G. Cesareni, Protein interaction data curation: the International Molecular Exchange (IMEx) consortium. Nature methods 9, 345-350 (2012).

4. S. Orchard, M. Ammari, B. Aranda, L. Breuza, L. Briganti, F. Broackes-Carter, N. H. Campbell, G. Chavali, C. Chen, N. Del-Toro, The MIntAct project-IntAct as a common curation platform for 11 molecular interaction databases. Nucleic acids research $\mathbf{4 2}$, D358-D363 (2014).

5. O. Keskin, N. Tuncbag, A. Gursoy, Predicting protein-protein interactions from the molecular to the proteome level. Chemical reviews 116, 4884-4909 (2016).

6. D. Szklarczyk, A. L. Gable, K. C. Nastou, D. Lyon, R. Kirsch, S. Pyysalo, N. T. Doncheva, M. Legeay, T. Fang, P. Bork, The STRING database in 2021: customizable protein-protein networks, and functional characterization of user-uploaded gene/measurement sets. Nucleic acids research 49, D605-D612 (2021).

7. A. J. Enright, I. Iliopoulos, N. C. Kyrpides, C. A. Ouzounis, Protein interaction maps for complete genomes based on gene fusion events. Nature 402, 86-90 (1999).

8. S. Saha, P. Chatterjee, S. Basu, M. Kundu, M. Nasipuri, FunPred-1: Protein function prediction from a protein interaction network using neighborhood analysis. Cellular and Molecular Biology Letters 19, 675-691 (2014).

9. L. R. Matthews, P. Vaglio, J. Reboul, H. Ge, B. P. Davis, J. Garrels, S. Vincent, M. Vidal, Identification of potential interaction networks using sequence-based searches for conserved protein-protein interactions or "interologs". Genome research 11, 2120-2126 (2001).

10. N. Bhardwaj, H. Lu, Correlation between gene expression profiles and protein-protein interactions within and across genomes. Bioinformatics 21, 2730-2738 (2005).

11. Q. Cong, I. Anishchenko, S. Ovchinnikov, D. Baker, Protein interaction networks revealed by proteome coevolution. Science 365, 185-189 (2019).

12. F. Pazos, D. Juan, J. M. Izarzugaza, E. Leon, A. Valencia, "Prediction of protein interaction based on similarity of phylogenetic trees" in Functional Proteomics (Springer, 2008), pp. 523-535.

13. S. Dong, V. Lau, R. Song, M. Ierullo, E. Esteban, Y. Wu, T. Sivieng, H. Nahal, A. Gaudinier, A. Pasha, Proteome-wide, structure-based prediction of protein-protein interactions/new molecular interactions viewer. Plant physiology 179, 1893-1907 (2019).

14. A. Baspinar, E. Cukuroglu, R. Nussinov, O. Keskin, A. Gursoy, PRISM: a web server and repository for prediction of protein-protein interactions and modeling their 3D complexes. Nucleic acids research 42, W285-W289 (2014).

15. M. N. Wass, G. Fuentes, C. Pons, F. Pazos, A. Valencia, Towards the prediction of protein interaction partners using physical docking. Molecular systems biology 7, 469 (2011).

16. B. Dai, C. Bailey-Kellogg, Protein interaction interface region prediction by geometric deep learning. Bioinformatics, (2021).

17. Q. C. Zhang, D. Petrey, J. I. Garzón, L. Deng, B. Honig, PrePPI: a structure-informed database of protein-protein interactions. Nucleic acids research 41, D828-D833 (2012).

18. D. Kuroda, J. J. Gray, Shape complementarity and hydrogen bond preferences in proteinprotein interfaces: implications for antibody modeling and protein-protein docking. Bioinformatics 32, 2451-2456 (2016). 
19. M. Y. Hein, N. C. Hubner, I. Poser, J. Cox, N. Nagaraj, Y. Toyoda, I. A. Gak, I. Weisswange, J. Mansfeld, F. Buchholz, A human interactome in three quantitative dimensions organized by stoichiometries and abundances. Cell 163, 712-723 (2015).

20. S. A. Ochsner, D. Abraham, K. Martin, W. Ding, A. McOwiti, W. Kankanamge, Z. Wang, K. Andreano, R. A. Hamilton, Y. Chen, The Signaling Pathways Project, an integrated 'omics knowledgebase for mammalian cellular signaling pathways. Scientific data 6, 1-21 (2019).

21. H. Wang, D. Chevalier, C. Larue, S. K. Cho, J. C. Walker, The protein phosphatases and protein kinases of Arabidopsis thaliana. The Arabidopsis Book/American Society of Plant Biologists 5, (2007).

22. C. Dardick, J. Chen, T. Richter, S. Ouyang, P. Ronald, The rice kinase database. A phylogenomic database for the rice kinome. Plant physiology 143, 579-586 (2007).

23. J. Choi, W. Lee, G. An, S.-R. Kim, OsCBE1, a Substrate Receptor of Cullin4-Based E3 Ubiquitin Ligase, Functions as a Regulator of Abiotic Stress Response and Productivity in Rice. International journal of molecular sciences 22, 2487 (2021).

24. K.-Y. Huang, J. T.-Y. Weng, T.-Y. Lee, S.-L. Weng, in BMC systems biology. (Springer, 2016), vol. 10, pp. 27-36.

25. X. Wang, Y. Li, M. He, X. Kong, P. Jiang, X. Liu, L. Diao, X. Zhang, H. Li, X. Ling, UbiBrowser 2.0: a comprehensive resource for proteome-wide known and predicted ubiquitin ligase/deubiquitinase-substrate interactions in eukaryotic species. Nucleic acids research, (2021).

26. H. Li, M. Wang, X. Xu, Prediction of kinase-substrate relations based on heterogeneous networks. Journal of bioinformatics and computational biology 13, 1542003 (2015).

27. G.-M. Qin, R.-Y. Li, X.-M. Zhao, PhosD: inferring kinase-substrate interactions based on protein domains. Bioinformatics 33, 1197-1204 (2017).

28. H. Wang, J. Qiu, H. Liu, Y. Xu, Y. Jia, Y. Zhao, HKPocket: human kinase pocket database for drug design. BMC bioinformatics 20, 1-11 (2019).

29. J. Gan, J. Qiu, C. Deng, W. Lan, Q. Chen, Y. Hu, Ksimc: Predicting kinase-substrate interactions based on matrix completion. International journal of molecular sciences $\mathbf{2 0}$, 302 (2019).

30. B. Xue, B. Jordan, S. Rizvi, K. M. Naegle, KinPred: A unified and sustainable approach for harnessing proteome-level human kinase-substrate predictions. PLoS computational biology 17, e1008681 (2021).

31. J. A. Ubersax, J. E. Ferrell Jr, Mechanisms of specificity in protein phosphorylation. Nature reviews Molecular cell biology 8, 530-541 (2007).

32. P. Jenardhanan, M. Panneerselvam, P. P. Mathur, Targeting kinase interaction networks: a new paradigm in PPI based design of kinase inhibitors. Current topics in medicinal chemistry 19, 467-485 (2019).

33. M. Huse, J. Kuriyan, The conformational plasticity of protein kinases. Cell 109, 275-282 (2002).

34. C. E. Berndsen, C. Wolberger, New insights into ubiquitin E3 ligase mechanism. Nature structural \& molecular biology 21, 301-307 (2014).

35. R. F. Alford, A. Leaver-Fay, J. R. Jeliazkov, M. J. O’Meara, F. P. DiMaio, H. Park, M. V. Shapovalov, P. D. Renfrew, V. K. Mulligan, K. Kappel, The Rosetta all-atom energy function for macromolecular modeling and design. Journal of chemical theory and computation 13, 3031-3048 (2017).

36. B. Tang, Z. Pan, K. Yin, A. Khateeb, Recent advances of deep learning in bioinformatics and computational biology. Frontiers in genetics 10, 214 (2019).

37. R. Kumari, S. K. Srivastava, Machine learning: A review on binary classification. International Journal of Computer Applications 160, (2017). 
38. K.-Y. Huang, H.-Y. Wu, Y.-J. Chen, C.-T. Lu, M.-G. Su, Y.-C. Hsieh, C.-M. Tsai, K.-I. Lin, H.-D. Huang, T.-Y. Lee, RegPhos 2.0: an updated resource to explore protein kinasesubstrate phosphorylation networks in mammals. Database 2014, (2014).

39. Y. Li, P. Xie, L. Lu, J. Wang, L. Diao, Z. Liu, F. Guo, Y. He, Y. Liu, Q. Huang, An integrated bioinformatics platform for investigating the human E3 ubiquitin ligasesubstrate interaction network. Nature communications 8, 1-9 (2017).

40. L. A. Kelley, S. Mezulis, C. M. Yates, M. N. Wass, M. J. Sternberg, The Phyre2 web portal for protein modeling, prediction and analysis. Nature protocols 10, 845-858 (2015).

41. S. Chaudhury, M. Berrondo, B. D. Weitzner, P. Muthu, H. Bergman, J. J. Gray, Benchmarking and analysis of protein docking performance in Rosetta v3. 2. PloS one 6, e22477 (2011).

42. Y. Yan, H. Tao, J. He, S.-Y. Huang, The HDOCK server for integrated protein-protein docking. Nature protocols 15, 1829-1852 (2020).

43. S. Basu, B. Wallner, DockQ: a quality measure for protein-protein docking models. PloS one 11, $\mathrm{e} 0161879$ (2016).

44. M. F. Lensink, R. Méndez, S. J. Wodak, Docking and scoring protein complexes: CAPRI 3rd Edition. Proteins: Structure, Function, and Bioinformatics 69, 704-718 (2007).

45. S. J. Baker, E. P. Reddy, CDK4: a key player in the cell cycle, development, and cancer. Genes \& cancer 3, 658-669 (2012).

46. F. Mercurio, H. Zhu, B. W. Murray, A. Shevchenko, B. L. Bennett, J. Wu Li, D. B. Young, M. Barbosa, M. Mann, A. Manning, IKK-1 and IKK-2: cytokine-activated IкB kinases essential for NF-kB activation. Science 278, 860-866 (1997).

47. S. Mitchell, J. Vargas, A. Hoffmann, Signaling via the NFкB system. Wiley Interdisciplinary Reviews: Systems Biology and Medicine 8, 227-241 (2016).

48. M. D. Daily, D. Masica, A. Sivasubramanian, S. Somarouthu, J. J. Gray, CAPRI rounds 3-5 reveal promising successes and future challenges for RosettaDock. PROTEINS:

Structure, Function, and Bioinformatics 60, 181-186 (2005).

49. H. Huang, B. M. Jedynak, J. S. Bader, Where have all the interactions gone? Estimating the coverage of two-hybrid protein interaction maps. PLoS computational biology $\mathbf{3}$, e214 (2007).

50. P. Blohm, G. Frishman, P. Smialowski, F. Goebels, B. Wachinger, A. Ruepp, D. Frishman, Negatome 2.0: a database of noninteracting proteins derived by literature mining, manual annotation and protein structure analysis. Nucleic acids research $\mathbf{4 2}$, D396-D400 (2014).

51. G. O. Consortium, The Gene Ontology (GO) database and informatics resource. Nucleic acids research 32, D258-D261 (2004).

52. E. H. Davidson, J. P. Rast, P. Oliveri, A. Ransick, C. Calestani, C.-H. Yuh, T. Minokawa, G. Amore, V. Hinman, C. Arenas-Mena, A genomic regulatory network for development. science 295, 1669-1678 (2002).

53. I. W. Mustika, H. N. Adi, F. Najib, in 2021 4th International Conference on Information and Communications Technology (ICOIACT). (IEEE, 2021), pp. 304-308.

54. M. Uzair, N. Jamil, in 2020 IEEE 23rd international multitopic conference (INMIC). (IEEE, 2020), pp. 1-6.

55. K. G. Sheela, S. N. Deepa, Review on methods to fix number of hidden neurons in neural networks. Mathematical problems in engineering 2013, (2013).

56. P. V. Hornbeck, J. M. Kornhauser, V. Latham, B. Murray, V. Nandhikonda, A. Nord, E. Skrzypek, T. Wheeler, B. Zhang, F. Gnad, 15 years of PhosphoSitePlus ${ }^{\circledR}$ : integrating post-translationally modified sites, disease variants and isoforms. Nucleic Acids Research 47, D433-D441 (2019). 
57. M. Varadi, S. Anyango, M. Deshpande, S. Nair, C. Natassia, G. Yordanova, D. Yuan, O. Stroe, G. Wood, A. Laydon, AlphaFold Protein Structure Database: Massively expanding the structural coverage of protein-sequence space with high-accuracy models. Nucleic acids research 50, D439-D444 (2022).

58. I. A. Vakser, Protein-protein docking: From interaction to interactome. Biophysical journal 107, 1785-1793 (2014).

59. J. Janin, K. Henrick, J. Moult, L. T. Eyck, M. J. Sternberg, S. Vajda, I. Vakser, S. J. Wodak, CAPRI: a critical assessment of predicted interactions. Proteins: Structure, Function, and Bioinformatics 52, 2-9 (2003).

60. M. F. Lensink, G. Brysbaert, T. Mauri, N. Nadzirin, S. Velankar, R. A. Chaleil, T. Clarence, P. A. Bates, R. Kong, B. Liu, Prediction of protein assemblies, the next frontier: The CASP14-CAPRI experiment. Proteins: Structure, Function, and Bioinformatics 89, 1800-1823 (2021).

61. A. Kryshtafovych, T. Schwede, M. Topf, K. Fidelis, J. Moult, Critical assessment of methods of protein structure prediction (CASP)—Round XIV. Proteins: Structure, Function, and Bioinformatics 89, 1607-1617 (2021).

62. M. D. Altman, E. A. Nalivaika, M. Prabu-Jeyabalan, C. A. Schiffer, B. Tidor, Computational design and experimental study of tighter binding peptides to an inactivated mutant of HIV-1 protease. Proteins: Structure, Function, and Bioinformatics 70, 678-694 (2008).

63. B. G. Pierce, K. Wiehe, H. Hwang, B.-H. Kim, T. Vreven, Z. Weng, ZDOCK server: interactive docking prediction of protein-protein complexes and symmetric multimers. Bioinformatics 30, 1771-1773 (2014).

64. M. A. Siddiq, G. K. Hochberg, J. W. Thornton, Evolution of protein specificity: insights from ancestral protein reconstruction. Current opinion in structural biology 47, 113-122 (2017).

65. K. A. Boehme, C. Blattner, Regulation of p53 Activity. Current Chemical Biology 4, 1-12 (2010).

66. N. A. Marze, S. S. Roy Burman, W. Sheffler, J. J. Gray, Efficient flexible backbone protein-protein docking for challenging targets. Bioinformatics 34, 3461-3469 (2018).

67. A. W. Senior, R. Evans, J. Jumper, J. Kirkpatrick, L. Sifre, T. Green, C. Qin, A. Žídek, A. W. Nelson, A. Bridgland, Improved protein structure prediction using potentials from deep learning. Nature 577, 706-710 (2020).

68. M. Baek, F. DiMaio, I. Anishchenko, J. Dauparas, S. Ovchinnikov, G. R. Lee, J. Wang, Q. Cong, L. N. Kinch, R. D. Schaeffer, C. Millán, H. Park, C. Adams, C. R. Glassman, A. DeGiovanni, J. H. Pereira, A. V. Rodrigues, A. A. v. Dijk, A. C. Ebrecht, D. J. Opperman, T. Sagmeister, C. Buhlheller, T. Pavkov-Keller, M. K. Rathinaswamy, U. Dalwadi, C. K. Yip, J. E. Burke, K. C. Garcia, N. V. Grishin, P. D. Adams, R. J. Read, D. Baker, Accurate prediction of protein structures and interactions using a three-track neural network. Science 373, 871-876 (2021).

69. J. Jumper, R. Evans, A. Pritzel, T. Green, M. Figurnov, O. Ronneberger, K.

Tunyasuvunakool, R. Bates, A. Žídek, A. Potapenko, Highly accurate protein structure prediction with AlphaFold. Nature 596, 583-589 (2021).

70. K. Vanommeslaeghe, E. Hatcher, C. Acharya, S. Kundu, S. Zhong, J. Shim, E. Darian, O. Guvench, P. Lopes, I. Vorobyov, CHARMM general force field: A force field for druglike molecules compatible with the CHARMM all-atom additive biological force fields. Journal of computational chemistry 31, 671-690 (2010).

71. S. A. McDowell, A simple derivation of the Boltzmann distribution. Journal of chemical education 76, 1393 (1999). 
72. D. B. Shear, The generalized Boltzmann distribution. Journal of theoretical biology 39, 165-169 (1973).

73. M. M. Lin, Generalized Boltzmann distribution for systems out of equilibrium. arXiv preprint arXiv:1610.02612, (2016).

74. E. Lounkine, M. J. Keiser, S. Whitebread, D. Mikhailov, J. Hamon, J. L. Jenkins, P. Lavan, E. Weber, A. K. Doak, S. Côté, Large-scale prediction and testing of drug activity on side-effect targets. Nature 486, 361-367 (2012).

75. L. Jia, Y. Sun, SCF E3 ubiquitin ligases as anticancer targets. Current cancer drug targets 11, 347-356 (2011).

76. M. M. Attwood, D. Fabbro, A. V. Sokolov, S. Knapp, H. B. Schiöth, Trends in kinase drug discovery: Targets, indications and inhibitor design. Nature Reviews Drug Discovery 20, 839-861 (2021).

77. P. Charbonneau, "C. RANDOM NUMBERS AND WALKS" in Natural Complexity (Princeton University Press, 2017), pp. 321-337.

78. W. L. DeLano, Pymol: An open-source molecular graphics tool. CCP4 Newsletter on protein crystallography 40, 82-92 (2002).

79. J. Choi, J.-H. Shin, H. J. An, M. J. Oh, S.-R. Kim, Analysis of secretome and Nglycosylation of Chlorella species. Algal Research 59, 102466 (2021).

80. J. Gutierrez, Metagenomic analysis of two soda lakes, with and without cyanobacterial bloom, with OmicsBox.

\section{Acknowledgments}

I appreciate Dr. Stefano Scopel at Sogang University for his advice on using deep learning as well as Dr. Doseok Kim at Sogang University, Soft-matter Optical Spectroscopy Laboratory in Sogang University, and Mr. Eunho Song at Seoul National University for their advice on data representation and identification of the missing explanation. Furthermore, I would like to thank Enago (www.enago.kr) for the English language review.

Funding: This study was conducted independently without any funding source.

Author contributions: This study was conducted by the sole author mentioned above.

Competing interests: The author declares no conflict of interest.

Data and materials availability: All data and codes, used in this study, are publicly available on GitHub (https://github.com/cjy318/protein_interaction) 
A
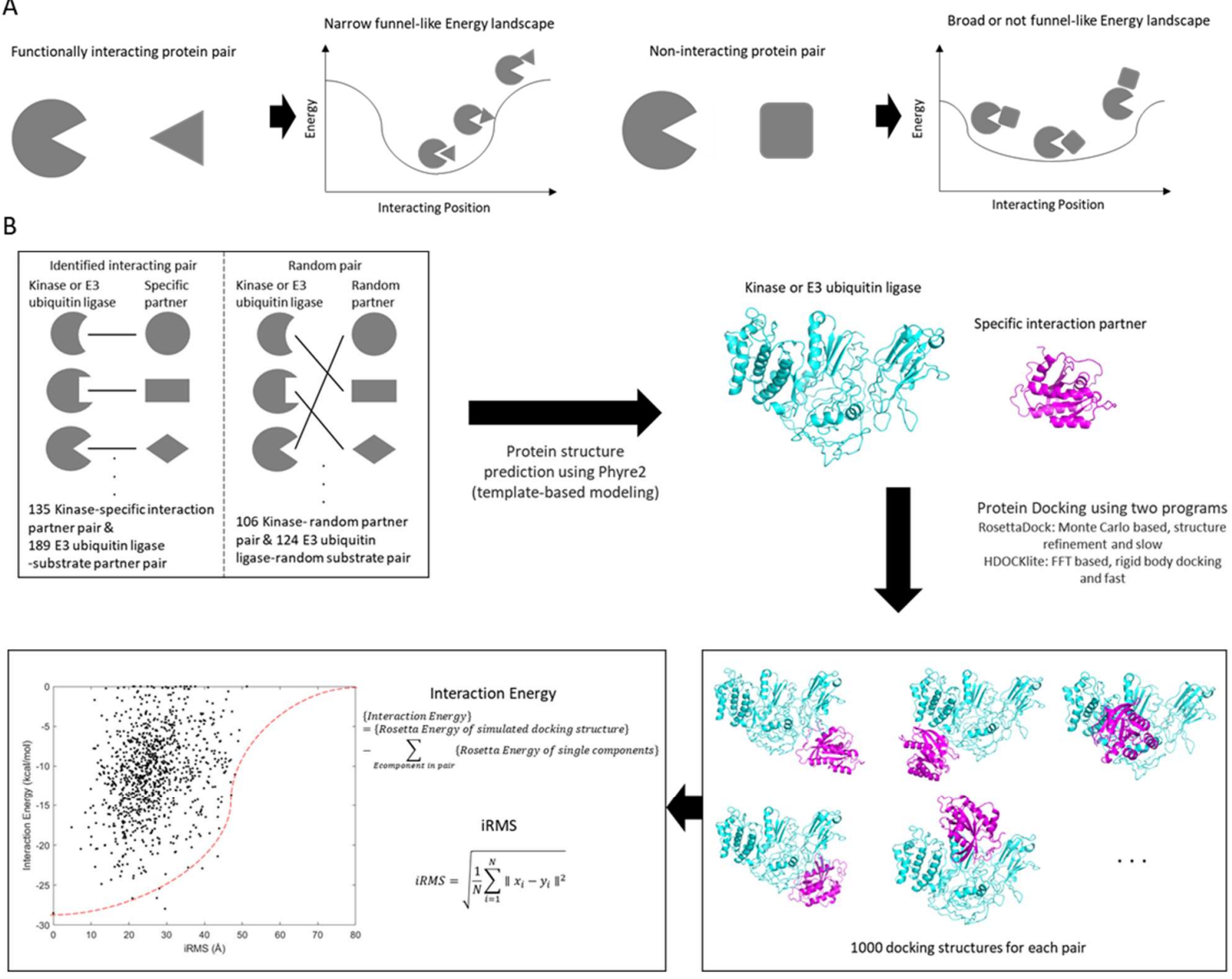

C

$$
\begin{aligned}
& \text { Strongest interacting structure } \\
& \text { (Reference structure) }
\end{aligned}
$$

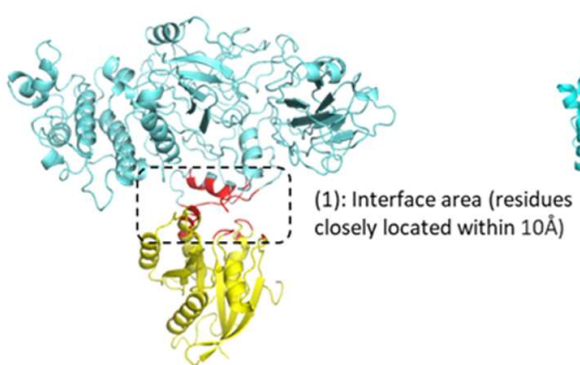

Another docking structure

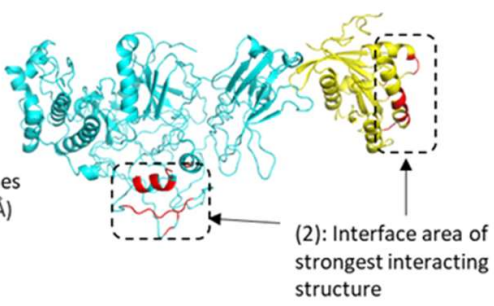

$$
i R M S=\sqrt{\frac{1}{N} \sum_{i=1}^{N}\left\|x_{i}-y_{i}\right\|^{2}}
$$

$N$ : \# atom in red region

$x_{i}$ : the atomic position of each atom in (1) $y_{i}:$ the atomic position of each atom in (2)

Figure 1. Narrow funnel-like interaction energy distribution hypothesis and workflow for interaction energy distribution analysis.

(A) Schematic diagram of narrow funnel hypothesis. Specific interactions might have narrow funnel-like interaction energy distribution. (B) Schematic diagram of interaction energy distribution analysis workflow. (C) Introduction of redefined iRMS. Residues of the interface area in the strongest interacting structure are colored in red. 
Table 1. Docking programs for possible docking structure generation.

\begin{tabular}{ccc}
\hline & RosettaDOCK & HDOCKlite \\
\hline Algorithm & Monte-Carlo & Fast-Fourier Transform \\
Scoring & Knowledge-based Energy Function & Shape-based Scoring Function \\
Structure Refinement & O & X (rigid body) \\
Speed & Slow* & Fast** $^{*}$ \\
Reference & $(41)$ & $(42)$ \\
\hline
\end{tabular}

${ }^{\star}$ For generation of 1,000 docking structures, several hours $\sim$ several days per interaction pair in personal computer (Intel@ Core i7-10700K $3.80 \mathrm{GHz}$ with 8 cores and $32 \mathrm{~GB}$ of RAM)

${ }^{\star \star}$ For generation of 1,000 docking structures, several minutes few hours per interaction pair in personal computer (Intel@ Core i7-10700K $3.80 \mathrm{GHz}$ with

8 cores and $32 \mathrm{~GB}$ of RAM) 

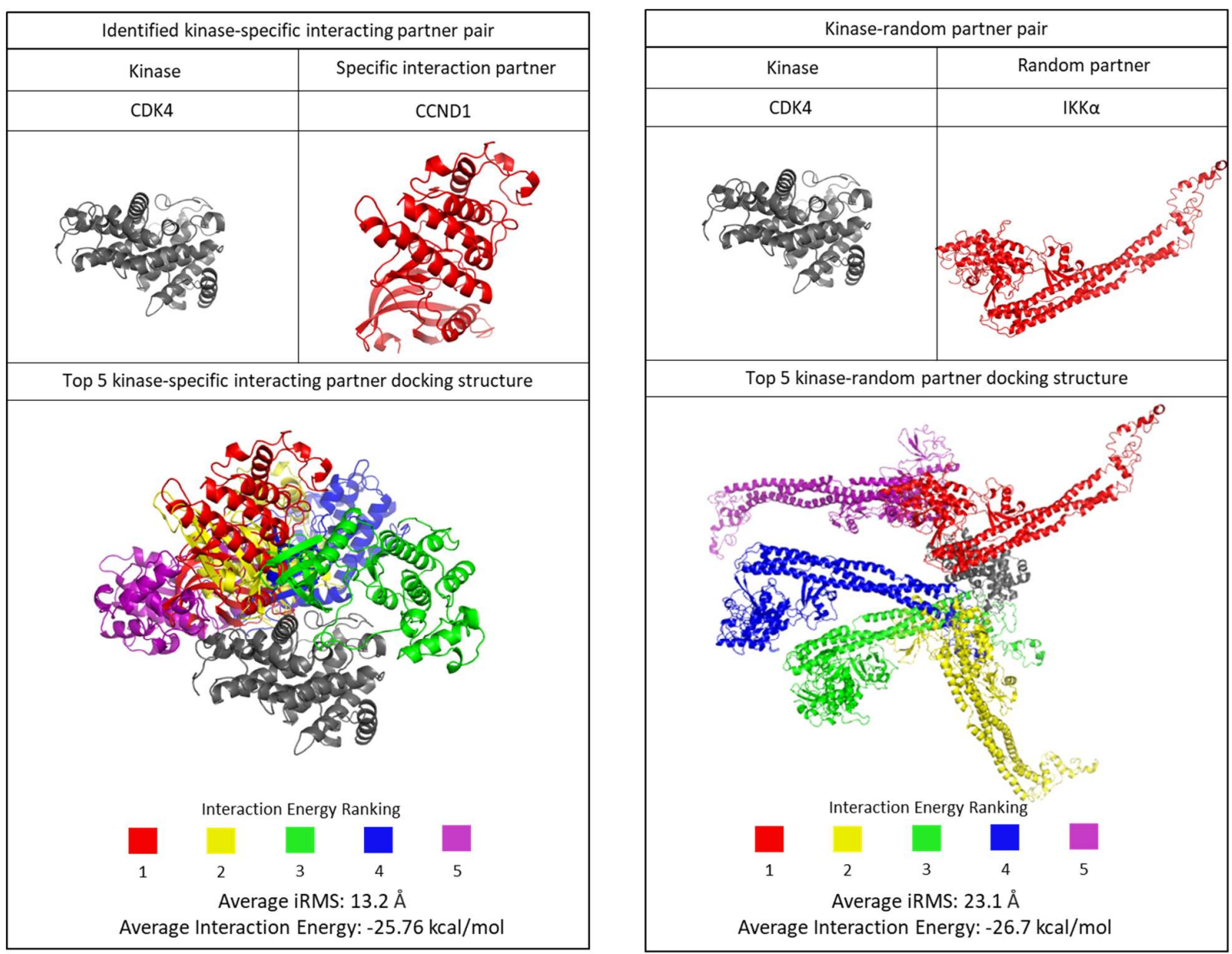

Figure 2. Structural meaning of narrow funnel-like interaction energy distribution.

The five strongest interacting structures of CDK4-CCND1 (identified specific interaction pair) and CDK4-IKK $\alpha$ (random pair). To avoid confusion, CDK4 in each pair is set in the same position and CDK4 in the strongest interacting structure is represented in gray. Each partner is colored in rainbow color in the order of interaction energy. 
A
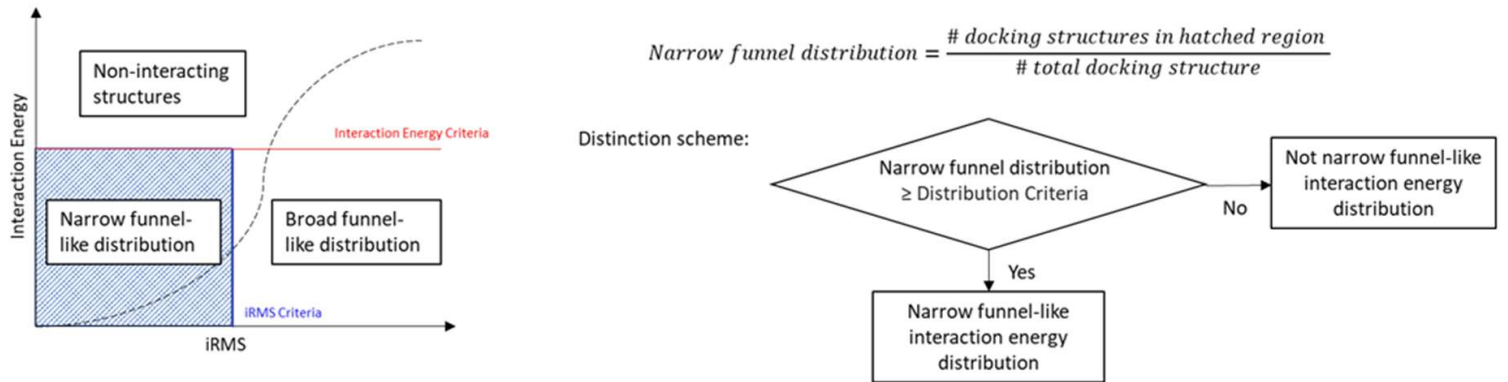

B
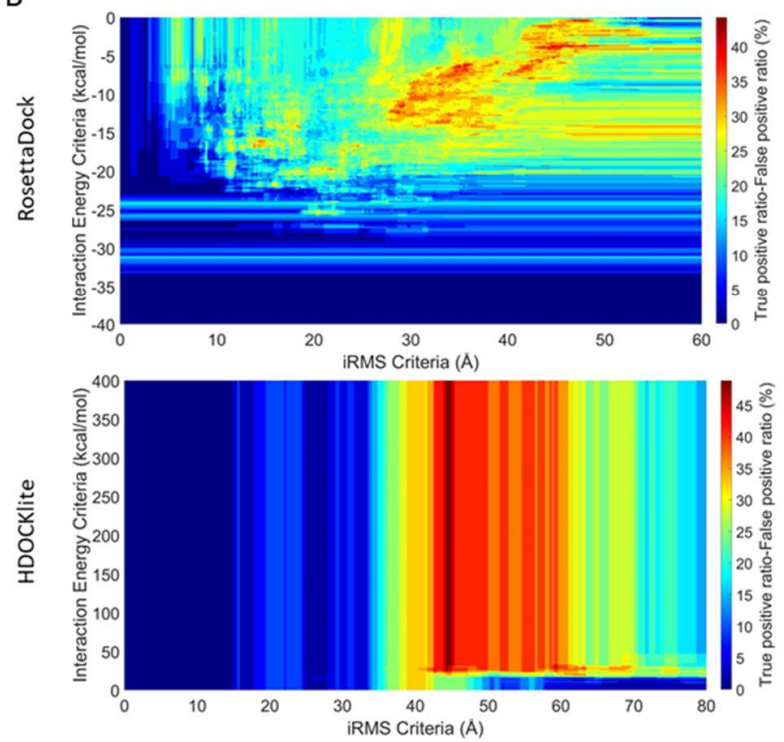

E3 ubiquitin ligase-substrate pair
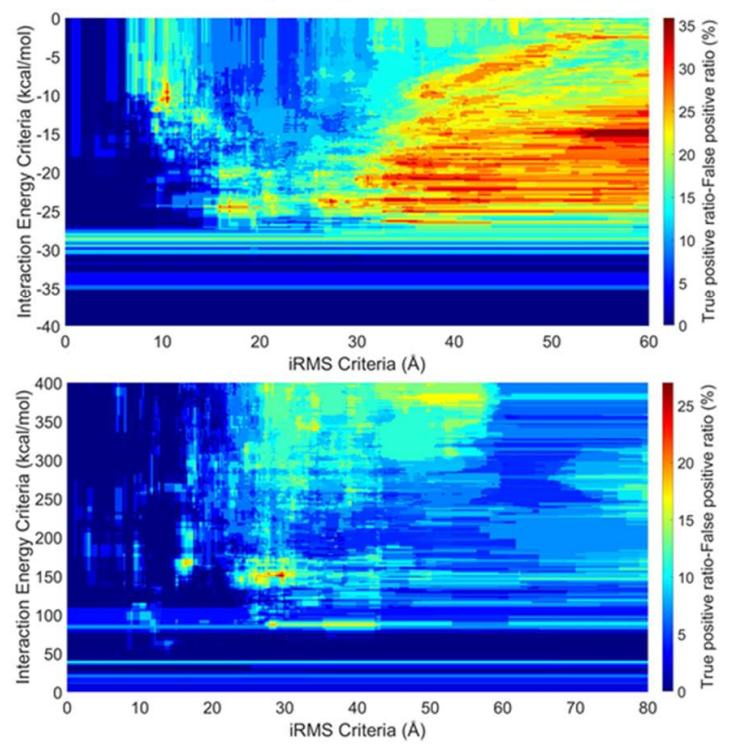
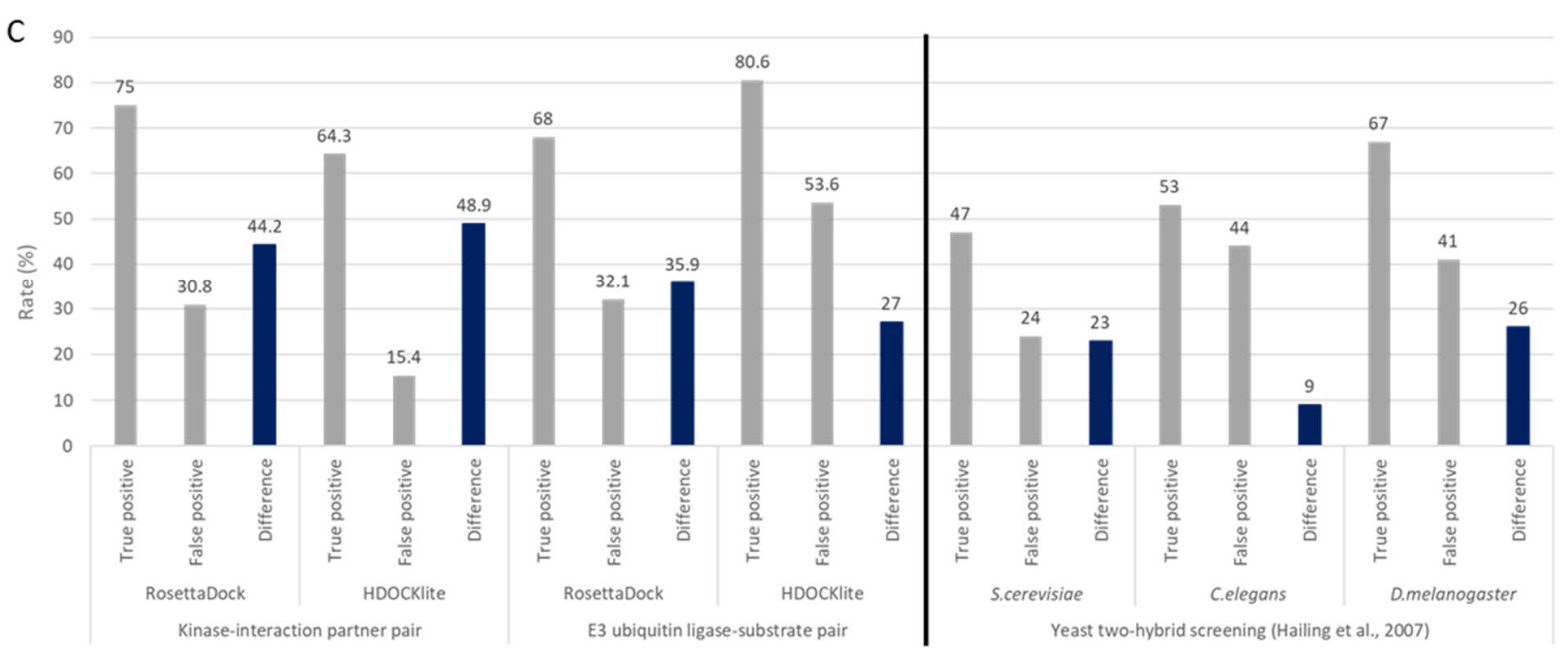

Figure 3. Quantitative analysis of narrow funnel-like interaction energy distribution and accuracy of predicting protein interactions by searching narrow funnel-like interaction distribution

(A) Scheme of the strategy to distinguish narrow funnel-like interaction energy distributions. (B) How well narrow funnel interaction energy distribution can distinguish specific interaction partners for each criteria. Among whole interaction pairs, pairs with high-accuracy prediction results $(>70 \%$ region with high confidence) and relatively small proteins ( $\leq 700$ residues) were analyzed. Difference ratios between true-positive (identified interacting protein complex with narrow funnel-like interaction energy distribution) and false-positive (random pair with narrow funnellike interaction energy distribution) for each criteria are shown on heatmap. Due to graphical representation limitations, only the maximum ratio differences are shown for each distribution criteria. (C) Rate comparisons for finding specific interaction partners using the narrow funnel and yeast two-hybrid screening methods. Comparison of genome-scale yeast two-hybrid analysis for three species (49). The criteria for finding narrow funnel-like energy distribution are described in Table 2. 
Table 2. Criteria to distinguish narrow funnel-like interaction energy distribution of interaction pairs with high-accuracy structure prediction results and relatively small proteins.

\begin{tabular}{ccccc}
\hline & \multicolumn{2}{c}{ RosettaDock } & \multicolumn{2}{c}{ HDOCKlite } \\
& Kinase pair & E3 ubiquitin ligase pair & Kinase pair & E3 ubiquitin ligase pair \\
\hline Interaction energy criteria $(\mathrm{kcal} / \mathrm{mol})$ & -3.6 & -23.4 & $26 \sim 400$ & $150 \sim 151$ \\
iRMS criteria $(\AA)$ & 44.6 & $39.6 \sim 40$ & 44.2 & $29.2 \sim 29.6$ \\
Distribution criteria $(\%)$ & 92.2 & 0.8 & 0.2 & 0.8 \\
\hline
\end{tabular}



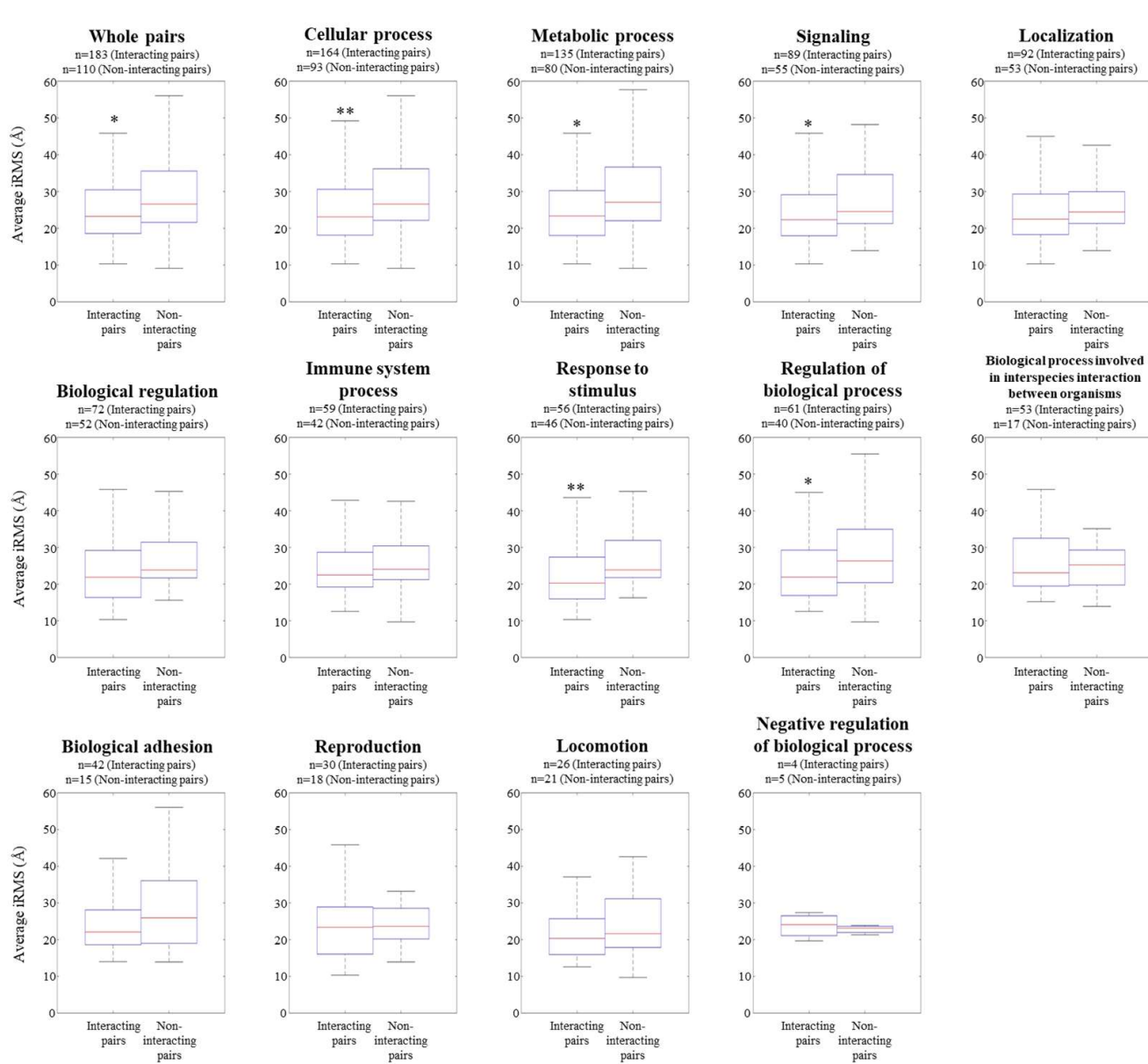

Developmenta

Figure 4. Distribution of average iRMS of interacting protein complexes and noninteracting protein pairs.

Distribution of average iRMSs of 1,000 docking structures of interacting protein complexes and noninteracting protein pairs are shown using box-and-whisker plot. Protein pairs divided according to the functional annotation with level-2 gene ontology distribution from Generic GO slim. Each related biological process in level-2 gene ontology from Generic GO slim and the number of protein pairs are notated. Significant differences of average iRMS with $95 \%$ confidence using Student's t-test are marked with asterisks and $99 \%$ confidence marked with double asterisks. 
Training Data
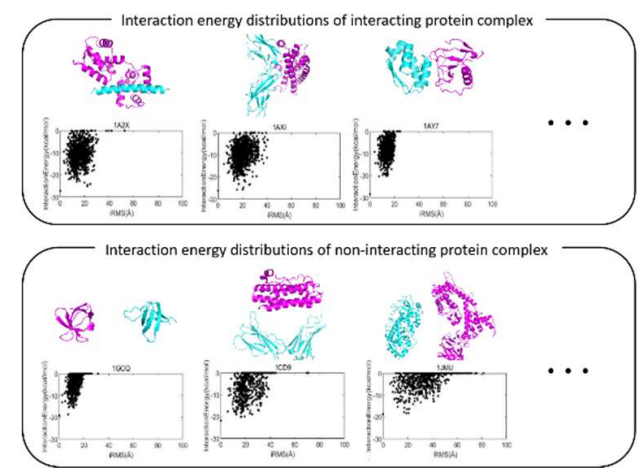

Deep learning

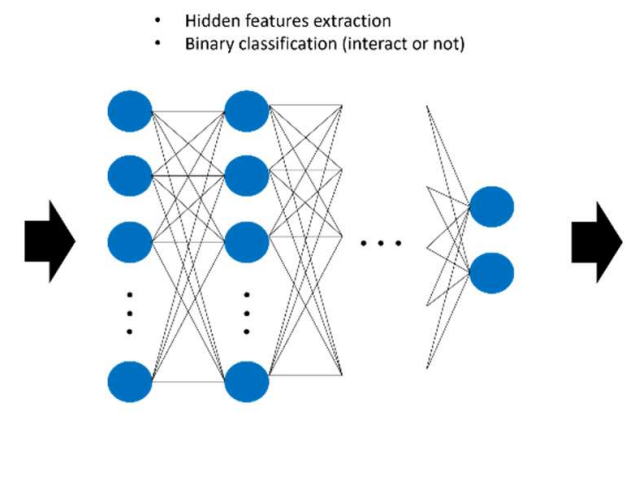

Prediction
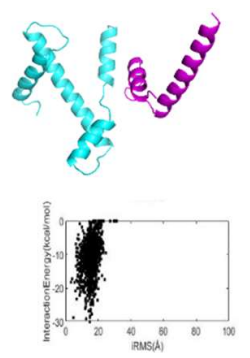

Do they interact or not?

Figure 5. Schematic diagram of protein interaction prediction using interaction energy distributions and deep learning.

This diagram describes the protein interaction prediction strategy based on the interaction energy diagram. 
1032

1033

1034

1035

1036

1037

1038

1039

1040

1041

1042

1043

1044

1045

1046

1047

1048

1049

Table 3. Highest accuracy of 10 -fold validation with data shuffling of candidate models for each protein interaction prediction and optimal deep learning model with the highest accuracy.

\begin{tabular}{|c|c|c|c|c|}
\hline & & General protein interaction prediction & Kinase substrate prediction & E3 ubiquitin ligase substrate prediction \\
\hline & ccuracy & $77.6 \%$ & $61.4 \%$ & $77.7 \%$ \\
\hline \multirow{3}{*}{$\begin{array}{c}\text { Optimal } \\
\text { model }\end{array}$} & Optimizer & Adamax & Adadelta & Adamax \\
\hline & Layer structure & $1024-64-2$ & $1024-64-2$ & $1024-2$ \\
\hline & Epochs & 109 & 9 & 61 \\
\hline
\end{tabular}




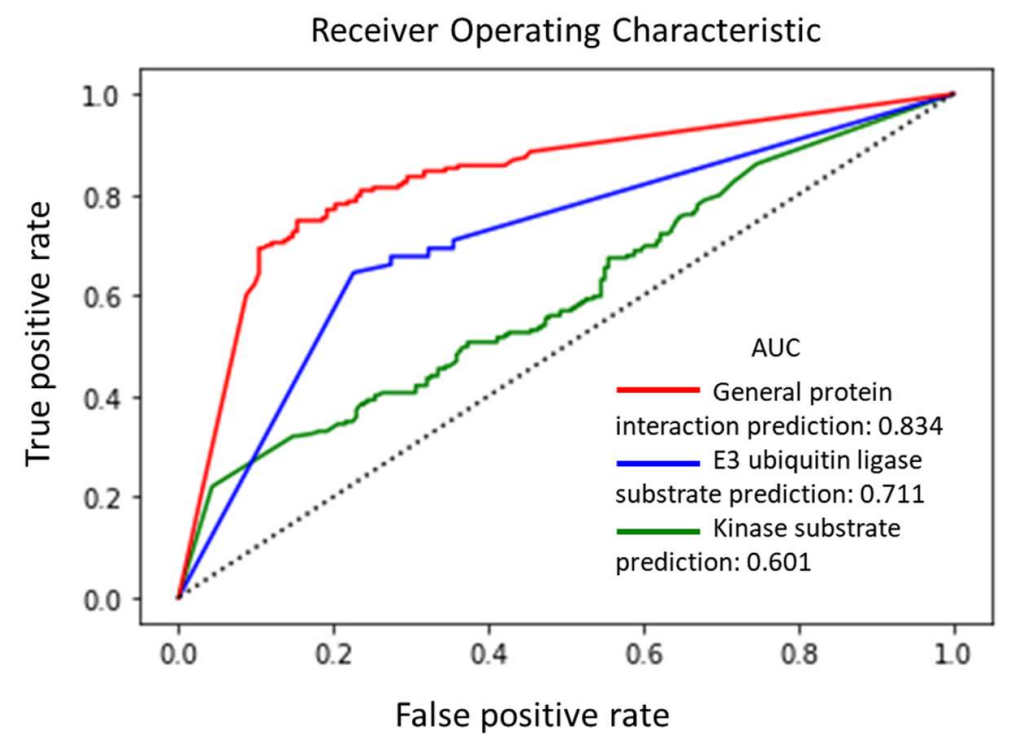

Figure 6. Predictive performance of deep learning models

Receiver operating characteristic (ROC) curves from 10-fold cross validation when shuffling data in the deep learning model to prediction protein interactions between E3 ubiquitin ligase and kinase substrates. Each area under curve (AUC) value is notated in the chart. 


\section{Supplementary Files}

This is a list of supplementary files associated with this preprint. Click to download.

- SupplementaryData1.xlsx

- SupplementaryData2.xlsx

- SupplementaryData3.xlsx

- SupplementaryData4.xlsx

- SupplementaryData5.xlsx

- SupplementaryData6.xlsx

- SupplementaryData7.xlsx

- SupplementaryData8.xlsx

- SupplementaryData9.xlsx

- SupplementaryFigure.pdf

- SupplementaryTables.pdf 